\title{
Evaluating the impact of point-of-care ultrasonography on patients with suspected acute heart failure or chronic obstructive pulmonary disease exacerbation in the emergency department: $A$ prospective observational study
}

Shunichiro Nakao (0, MD, MSc ${ }^{*}$; Christian Vaillancourt, MD, MSc ${ }^{\ddagger \S}$; Monica Taljaard, $\mathrm{PhD}^{*}$;

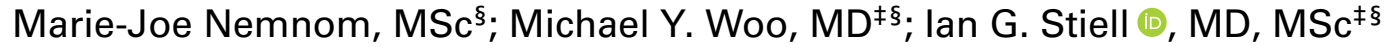

\begin{abstract}
CLINICIAN'S CAPSULE
What is known about the topic?

Lung point-of-care ultrasonography (POCUS) for the assessment of shortness of breath has high sensitivity and specificity for identifying pulmonary edema.

What did this study ask?

What is the clinical impact of POCUS in emergency department (ED) patients with suspected acute heart failure or chronic obstructive pulmonary disease (COPD)? What did this study find?

Patients evaluated with lung POCUS had no difference in length of stay in ED but received appropriate treatment faster. Why does this study matter to clinicians?

Lung POCUS could result in faster treatments for patients with suspected acute heart failure and COPD.
\end{abstract}

\section{ABSTRACT}

Objectives: Acute heart failure and chronic obstructive pulmonary disease (COPD) are sometimes difficult to differentiate in the emergency department (ED). We sought to determine the clinical impact of point-of-care ultrasonography (POCUS) in ED patients with suspected acute heart failure or COPD.

Methods: We conducted a prospectively collected cohort study with health records review with frequency matching at The Ottawa Hospital between March and September 2017. We included patients aged 50 and older with shortness of breath or cough from suspected acute heart failure or COPD. Our primary outcome was ED length of stay. Secondary outcomes were time to disposition decision, time to appropriate treatment, and the incidence of adverse events. We analyzed time-to-event outcomes using Kaplan-Meier analysis and Cox regression analysis with POCUS analyzed as a timedependent variable, and the incidence of adverse events using logistic regression analyses.

Results: There were 81 patients evaluated with lung POCUS and 243 matched patients who were not. Lung POCUS was not significantly associated with ED length of stay and time to disposition decision; however, patients evaluated with lung POCUS received disease-specific treatment faster compared with the non-POCUS group (adjusted hazard ratio, 1.50 [95\% confidence interval, 1.05-2.15], a median time difference of 31 minutes). We found no significant differences in the incidence of adverse events.

Conclusions: In this study, use of lung POCUS resulted in no difference in ED length of stay and time to disposition decision, but was associated with faster administration of diseasespecific treatments for elderly patients with suspected acute heart failure or COPD.

\section{RÉSUMÉ}

Objectif: Il est parfois difficile de distinguer l'insuffisance cardiaque aiguë (ICA) et la maladie pulmonaire obstructive chronique (MPOC) I'une de l'autre au service des urgences (SU). Aussi l'étude visait-elle à déterminer la portée clinique de l'échographie au chevet (EC) chez les patients examinés au SU pour une présomption d'ICA ou de MPOC.

Méthode: Il s'agit d'une étude de cohortes, prospective, consistant en un examen de dossiers médicaux et en un appariement de patients fondé sur la fréquence, menée à L'Hôpital d'Ottawa, entre mars et septembre 2017. Étaient retenus les patients de 50 ans et plus, présentant de l'essoufflement ou de la toux secondaires à une ICA ou à une MPOC présumées. Le principal critère

From the * University of Ottawa, Ottawa, ON; ${ }^{\dagger}$ Department of Traumatology and Acute Critical Medicine, Osaka University Graduate School of Medicine, Suita, Japan; ${ }^{\ddagger}$ Department of Emergency Medicine, University of Ottawa, Ottawa, ON; and the ${ }^{\S}$ Ottawa Hospital Research Institute, Ottawa, ON.

Correspondence to: Dr. Shunichiro Nakao, Department of Traumatology and Acute Critical Medicine, Osaka University Graduate School of Medicine, 2-15 Yamadaoka, Suita, Osaka, Japan; Email: shunichironakao@ hp-emerg.med.osaka-u.ac.jp 
d'évaluation était la durée du séjour au SU. Les critères d'évaluation secondaires comprenaient le temps écoulé avant la prise de décision quant aux suites à donner et avant la mise en route du traitement approprié, ainsi que l'incidence d'événements indésirables. Le temps écoulé avant les événements a été calculé selon la méthode de Kaplan-Meier et l'analyse de régression de Cox, où l'EC était considérée comme une variable dépendante du temps; et l'incidence des événements indésirables, déterminée par des analyses de régression logistique.

Résultats: Au total, 81 patients ont été soumis à une EC pulmonaire et 243 patients appariés ne l'ont pas été. L'EC pulmonaire n'a pas été associée de manière significative à la durée du séjour au SU ni au temps écoulé avant la prise de décision quant aux suites à donner; par contre, les patients soumis à une EC pulmonaire ont reçu un traitement approprié plus rapidement que ceux qui ne l'avaient pas été (rapport de risque rajusté : 1,50 [IC à 95\% : 1,05-2,15]; écart médian : 31 minutes). Aucun écart significatif n'a été relevé quant à l'incidence d'événements indésirables.

Conclusion: II ressort de l'étude que le recours à l'EC pulmonaire ne s'est pas traduit par une différence de la durée du séjour au SU ni du temps écoulé avant la prise de décision quant aux suites à donner; par contre, il a été associé à une mise en route plus rapide des traitements appropriés chez les personnes âgées souffrant d'une ICA ou d'une MPOC présumées.

Keywords: Acute heart failure, emergency medicine, point-ofcare ultrasonography, shortness of breath

\section{INTRODUCTION}

Acute heart failure and exacerbation of chronic obstructive pulmonary disease (COPD) are common causes of shortness of breath, and are often not easy to differentiate in the emergency department (ED). ${ }^{1-3}$ The clinical symptoms are often not specific to one condition or the other, and there is no standardized clinical criteria to differentiate them in the ED. ${ }^{4,5}$ A complete assessment including laboratory results often takes more than 40 minutes. ${ }^{6,7}$ As a result, patients are often treated for both heart failure and COPD, and then re-evaluated to assess initial treatment responses. ${ }^{2}$

Lung POCUS can identify acute heart failure and lung congestion by the number of B-lines with a high sensitivity and specificity as shown in recent systematic reviews. ${ }^{8,9}$ Although lung POCUS is suggested in the European guidelines, it is not currently considered standard care for the diagnosis of acute heart failure. ${ }^{10}$ Moreover, the time effectiveness and clinical impact of lung POCUS has not yet been reported.

The overall objective of this study is to determine the clinical impact of lung POCUS on ED length of stay which is an important patient-oriented outcome. We also aimed to assess time to disposition decision, time to appropriate treatment administration, and the incidence of adverse events.

\section{METHODS}

\section{Study design}

We performed a prospectively collected cohort study with health records review comparing matched cohorts
(1:3) of patients who were and were not exposed to lung POCUS. This study was approved by the local Research Ethics Board (REB) on March 16, 2017.

\section{Study setting and population}

The study was conducted at the Ottawa Hospital, General and Civic campuses in Ottawa, Canada. We included patients who were assessed for shortness of breath or cough, were 50 years of age or older, and had suspected diagnoses of acute heart failure or COPD exacerbation by ED physicians. We considered documented ED diagnoses in physician records as suspected diagnoses in the ED. We excluded patients diagnosed with acute ST-segment elevation myocardial infarction on arrival electrocardiogram. We also excluded those who had known history of interstitial fibrosis, extensive lung cancer, pneumonectomy or lobectomy, or pneumothorax.

For each patient with lung POCUS, we only included recorded lung POCUS performed with a clinical indication. We did not include purely educational scans as determined from the POCUS record. We selected a consecutive sample of matched patients without lung POCUS using the following matching criteria: 5-year age strata, sex, and with or without a previous diagnosis of heart failure and/or COPD.

\section{Study protocol}

Lung POCUS was performed as part of usual care. We used an eight-zone technique, and each scan was deemed to be positive for pulmonary edema when at least three 
B-lines were present in more than two zones per side. ${ }^{11-}$ ${ }^{13}$ All POCUS scans were reviewed by the Emergency Medicine Ultrasonography team to confirm findings.

We used the following hierarchical criteria to define the reference standard: (1) a discharge diagnosis for admitted patients, (2) an ED diagnosis with a repeat ED visit or a follow-up visit to outpatient clinic for the same initially presumed diagnosis within a month after the first ED visit, or (3) in all other cases where a diagnosis was only made by an ED physician, study investigators reviewed health records of the ED care independently and determined the final diagnosis by consensus.

We collected standardized information on patient demographics from triage notes, ED health records by ED physicians, nursing documentations, and radiological reports.

\section{Measures}

For evaluating ED length of stay as a priori defined primary outcome, we obtained the time of registration to the ED, and the time the patient left the ED, which was the moment of the admission or the time when the patient physically left the ED regardless of destination. ED length of stay was defined as the interval between these times. The time of POCUS was defined using the automatically recorded time when the first lung image for assessing B-lines was archived.

For evaluating our secondary outcome, namely time to disposition decision, we obtained the time of disposition, which was the time when ED physicians or consultants make the decision about the patient's disposition, such as to discharge or admit the patient. We defined time to disposition decision as the time interval between the time of physician initial assessment and the time of disposition decision of discharge or admission.

For evaluating the secondary outcome of time to disease-specific treatment administration in the ED, patients were classified as receiving appropriate treatment when the final diagnosis in the ED was matched with the disease-specific treatment. We defined time to disease-specific treatment administration as the time interval between the time of physician initial assessment and the time of disease-specific appropriate treatment administration. Disease-specific treatment for acute heart failure was defined as diuretic administration, intravenous vasodilator administration, or emergency dialysis. Disease-specific treatment for COPD exacerbation was defined as administration of bronchodilator, such as beta-agonist or anticholinergic, or inhaled or systemic steroid administration. We did not count oxygen administration and any types of positive pressure ventilation as disease-specific therapy because they can be used for acute heart failure as well as COPD exacerbation.

The incidence of adverse events was another a priori defined secondary outcome. We defined adverse events as: (1) potentially unnecessary treatment administration; (2) return ED visits within 7 days after initial ED visit; and (3) inaccurate diagnosis in the ED. The potentially unnecessary treatment administration was defined as the disease-specific treatment administered in the ED inconsistent with the reference standard diagnosis. We also recorded for each patient whether they returned to the Ottawa Hospital ED with a related respiratory complaint within 7 days after the initial visit. An inaccurate diagnosis was defined as ED diagnosis inconsistent with the final diagnosis by the reference standard.

\section{Data analysis}

We described differences between the groups with and without lung POCUS using medians, means, and proportions, and assessed statistical significance of the differences using Mann-Whitney U-test or Student t-test for continuous variables, or Fisher's exact test for categorical variables. Cohen's kappa was used to assess inter-rater agreement for the lung POCUS interpretations by study investigators.

We described the median times to event for patients with and without POCUS using Kaplan-Meier analysis, treating lung POCUS as a time-dependent variable to account for the fact that lung POCUS was administered at some time after registration in the ED. To evaluate the association of lung POCUS with ED length of stay, time to disposition decision, and time to disease-specific treatment, we used Cox proportional hazards regression analysis. The use of lung POCUS was the primary exposure of interest, and was modeled as a time-dependent variable. ${ }^{14}$ This means that the value of lung POCUS was 0 if lung POCUS had not been performed, and it turned to 1 only after it was performed. Therefore, patients who received appropriate treatment before lung POCUS contributed their time before treatment as unexposed, similar to patients who never received lung POCUS. The effect of lung POCUS was expressed as an adjusted hazard ratio (HR) together with a $95 \%$ confidence interval (CI). An HR greater than 1 meant that lung POCUS shortened the length of time until the event. We adjusted 
for any residual baseline imbalance after matching between the groups by entering variables identified as being statistically significantly different between the two groups at $\alpha=0.2$ as covariates into the Cox models. We assessed the proportional hazard assumption using graphical and numerical tests based on martingale residuals. ${ }^{15}$ For the variables which did not satisfy the proportional hazard assumption, we included interaction terms between the covariates and time in the model; this allowed the effect of the relevant variables to change over time. A priori primary outcome measure was analyzed as per the initial REB application and study protocol.

To examine differences in adverse events between the two groups, we calculated unadjusted and adjusted odds ratio (OR) by using exact logistic regression analyses, adjusting for variables considered to be clinically important. We used SAS 9.4 (Cary, NC, USA) for all statistical analyses.

\section{RESULTS}

\section{Study patients}

Figure 1 summarizes the inclusion and exclusion of patients. Among 3,538 patients aged 50 or older who visited the ED with shortness of breath or cough as a chief complaint during the study period, 81 (2.3\%) were included in the lung POCUS group. After the 1:3 matching process, 243 patients among the remaining group were included. The characteristics of the POCUS and matched control patients are summarized in Table 1 and Supplemental Table S1. The median age of the total cohort was 79 years. Most signs, symptoms, and findings in the ED were similar between the groups. Inhaled beta-agonists were rarely nurse-initiated and administered before physician assessment took place. This was the case for three $(3.7 \%)$ patients in the POCUS group and $8(3.3 \%)$ in the comparison group, and all of them had an existing prescription for inhaled bronchodilators. All of these treatments were considered to be appropriate, and most of them were administered within 30 minutes from triage. These 11 patients were excluded for the time-to-event analyses of appropriate treatment because the event happened before time 0 (physician initial assessment time). Using our described reference standard, $64(79.0 \%)$ patients in the lung POCUS group and $163(67.1 \%)$ in the comparison group were diagnosed with acute heart failure. There was substantial inter-rater agreement on ED diagnosis by study investigators as demonstrated by Cohen's kappa of 0.79 .

The characteristics of lung POCUS and the timing of POCUS with the timeline of ED care are presented in Supplemental Table S2 and Figure S1. There was substantial inter-rater agreement as demonstrated by Cohen's kappa of 0.80 for the reviewed interpretations of lung POCUS by study investigators.

\section{Main results}

Unadjusted and adjusted HRs from the Cox regression analyses are presented in Table 2. Lung POCUS was not significantly associated with ED length of stay (adjusted HR, 1.11 [95\% CI, 0.85-1.46], $p=0.44$ ) and time to disposition decision (adjusted HR, 1.05 [95\% CI, 0.79-1.40], $p=0.73$ ). That said, patients evaluated with lung POCUS received disease-specific treatment faster (adjusted HR, 1.50 [95\% CI, 1.05-2.15], $p=0.03$ ). The Kaplan-Meier analysis, which accounted for the time-dependent nature of POCUS, showed that the median time to disease-specific treatment administration was shorter in the lung POCUS group than in the comparison group (61 v. 92 minutes; Table 2; Supplemental Figure S2).

After the multivariable adjustment, we found no significant difference in administration of potentially unnecessary treatment or unscheduled ED visits within 7 days. However, we observed a lower incidence of inaccurate ED diagnosis in the lung POCUS group (adjusted OR, 0.25 [95\% CI, 0-1.24], $p=0.08$ ). Analyses of the incidence of adverse events are presented in Supplemental Table S3 and S4. There were eight patients (9.9\%) who were treated for both acute heart failure and COPD exacerbation in the lung POCUS group, $33(13.6 \%)$ in the comparison group, three of which were considered as truly having both diseases.

\section{DISCUSSION}

We did not observe a statistically significant association between ED length of stay or time to disposition decision and use of lung POCUS among patients with suspected acute heart failure or COPD. However, the adjusted HR suggests the use of lung POCUS was associated with faster median administration time of disease-specific treatments for acute heart failure and COPD patients by 


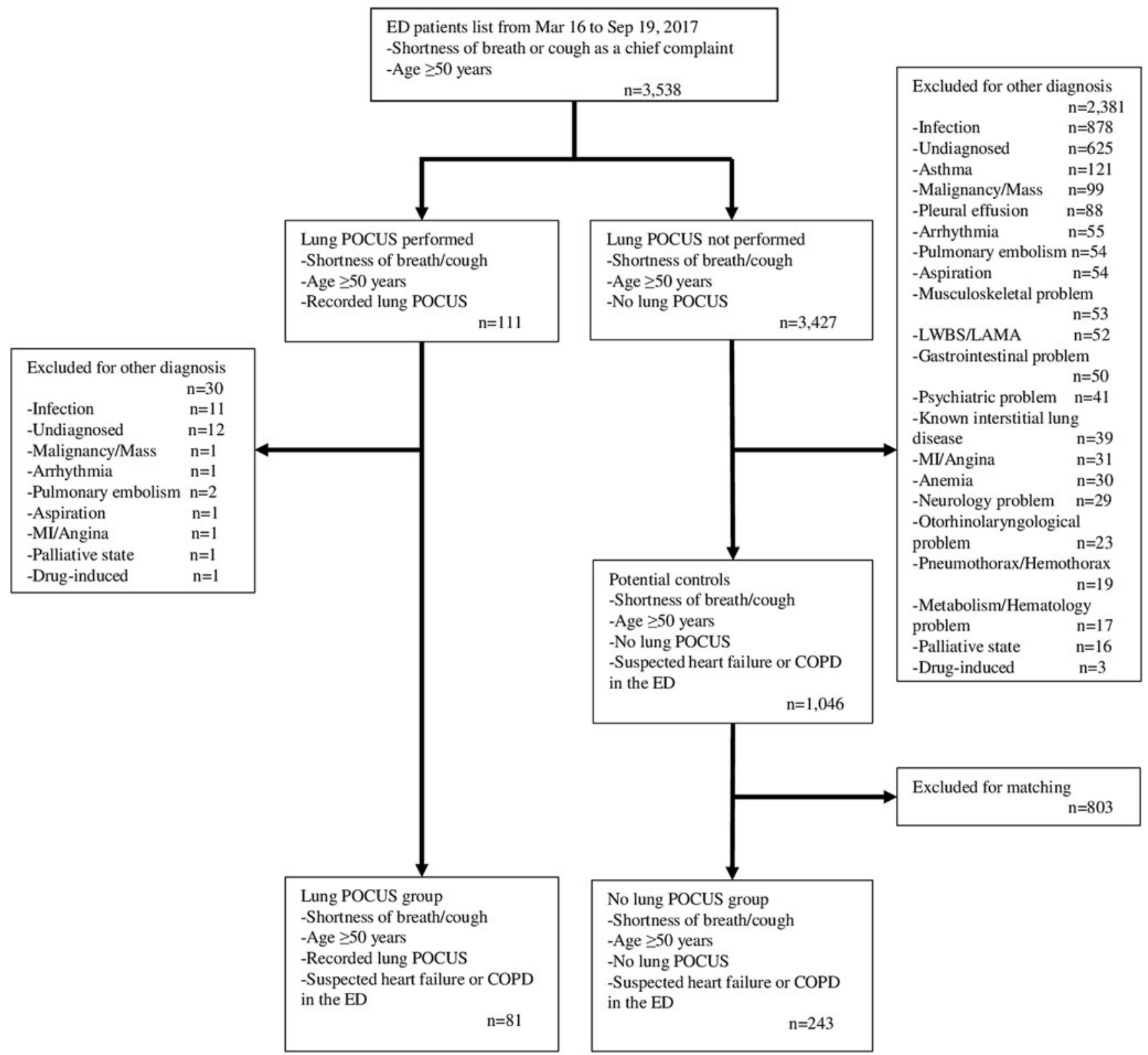

Figure 1. Patient flow. $E D=$ emergency department; POCUS = point-of-care ultrasonography; $C O P D=$ chronic obstructive pulmonary disease; LWBS = left without being seen; LAMA = left against medical advice.

31 minutes. We did not find statistically significant differences in the incidence of predefined adverse events, although there was a tendency of having less inappropriate ED diagnosis in the lung POCUS group.

To our knowledge, no other study has been published specifically examining the ability of lung POCUS to decrease ED length of stay among adult dyspneic patients. However, several studies suggest POCUS could decrease ED length of stay for other medical conditions. ${ }^{16-18}$ A randomized trial of pediatric population showed a statistically significant decrease in ED length of stay by using lung POCUS for diagnosing pneumonia. ${ }^{19}$

We found a statistically significant reduction in the time to appropriate treatment administration associated with lung POCUS. The potential benefits of a reduction in time include early relief of symptoms as well as early prognosis. Matsue and colleagues found that faster administration of diuretics for patients with acute heart failure might be associated with decreased in-hospital 


\begin{tabular}{|c|c|c|c|c|c|}
\hline \multirow[b]{2}{*}{ Characteristics } & \multicolumn{2}{|c|}{ Lung POCUS group } & \multicolumn{2}{|c|}{ No lung POCUS group } & \multirow[b]{2}{*}{$p$-Value } \\
\hline & \multicolumn{2}{|c|}{$n=81$} & \multicolumn{2}{|c|}{$n=243$} & \\
\hline Age, median, Q1-03 & 79 & $73-86$ & 79 & $73-86$ & 0.97 \\
\hline Female sex, $\mathrm{n}(\%)$ & 41 & $(50.6)$ & 123 & $(50.6)$ & 1.00 \\
\hline Past history of heart failure and/or COPD, $\mathrm{n}(\%)$ & & & & & 1.00 \\
\hline Heart failure only & 29 & (35.8) & 87 & (35.8) & \\
\hline COPD only & 11 & $(13.6)$ & 33 & $(13.6)$ & \\
\hline Heart failure and COPD & 16 & (19.8) & 48 & (19.8) & \\
\hline No heart failure or COPD & 25 & $(30.9)$ & 75 & (30.9) & \\
\hline Arrival by ambulance, $\mathrm{n}(\%)$ & 42 & $(51.9)$ & 130 & $(53.5)$ & 0.80 \\
\hline CTAS, median, Q1-Q3 & 2 & $2-3$ & 2 & $2-3$ & 0.28 \\
\hline \multicolumn{6}{|l|}{ Vital signs on arrival, mean (SD) } \\
\hline Systolic BP (mmHg) & 139.7 & (29.3) & 140.3 & $(25.1)$ & 0.87 \\
\hline Heart rate (/minute) & 87.8 & $(23.5)$ & 89.4 & $(21.9)$ & 0.58 \\
\hline Body temperature (Celsius) & 36.2 & $(0.82)$ & 36.4 & $(0.83)$ & $\underline{0.13}$ \\
\hline Respiration rate (/minute) & 23.7 & $(7.3)$ & 22.9 & $(6.0)$ & 0.37 \\
\hline Oxygen saturation (\%) & 92.0 & $(6.7)$ & 92.6 & $(5.5)$ & 0.44 \\
\hline Chest X-ray, n (\%)* & 81 & $(100)$ & 240 & (98.8) & 0.58 \\
\hline Fluid congestion on X-ray $(n=321)$ & 43 & $(53.1)$ & 117 & $(48.2)$ & 0.45 \\
\hline Medicine administered in ED, $\mathrm{n}(\%)$ & 74 & $(91.4)$ & 218 & (89.7) & 0.83 \\
\hline \multicolumn{6}{|l|}{ Disease-specific treatment for heart failure } \\
\hline Diuretics & 60 & $(74.1)$ & 149 & (61.3) & 0.04 \\
\hline Vasodilators & 12 & $(14.8)$ & 41 & (16.9) & 0.73 \\
\hline Emergency dialysis & 1 & $(1.2)$ & 5 & $(2.1)$ & 1.00 \\
\hline \multicolumn{6}{|l|}{ Disease-specific treatment for COPD } \\
\hline Beta agonists & 19 & $(23.5)$ & 80 & (32.9) & 0.13 \\
\hline Anticholinergics & 17 & $(21.0)$ & 70 & $(28.8)$ & 0.19 \\
\hline Steroids & 12 & $(14.8)$ & 61 & $(25.1)$ & 0.07 \\
\hline $\begin{array}{l}\text { Disease-specific treatment administered } \\
\text { before physician initial assessment, } \mathrm{n}(\%)\end{array}$ & 3 & $(3.7)$ & 8 & (3.3) & 1.00 \\
\hline $\begin{array}{l}\text { Disease-specific treatment administered after physician } \\
\text { initial assessment, before lung POCUS, } n(\%)\end{array}$ & 26 & $(32.1)$ & - & & - \\
\hline Initial diagnosis in ED, $\mathrm{n}(\%)$ & & & & & 0.14 \\
\hline Acute heart failure & 64 & $(79.0)$ & 168 & $(69.1)$ & \\
\hline COPD & 14 & $(17.3)$ & 68 & $(28.0)$ & \\
\hline Acute heart failure and COPD & 3 & (3.7) & 7 & $(2.9)$ & \\
\hline Disposition, n (\%) & & & & & 0.80 \\
\hline Discharged home & 34 & $(42.0)$ & 108 & $(44.4)$ & \\
\hline Admitted to hospital & 47 & $(58.0)$ & 135 & $(55.6)$ & \\
\hline Final diagnosis, $n(\%) \dagger$ & & & & & 0.17 \\
\hline Acute heart failure & 64 & $(79.0)$ & 163 & $(67.1)$ & \\
\hline COPD & 14 & (17.3) & 66 & $(27.2)$ & \\
\hline Acute heart failure and COPD & 3 & $(3.7)$ & 9 & $(3.7)$ & \\
\hline Other & 0 & (0) & 5 & (2.1) & \\
\hline
\end{tabular}

mortality. ${ }^{20}$ Therefore, our finding of a 31-minute reduction of time to appropriate treatment can be clinically significant.
No other studies have found a significant effect of POCUS on adverse events. In a previous study, $14.3 \%$ of patients who were admitted with acute decompensated 


\begin{tabular}{|c|c|c|c|c|c|c|}
\hline \multirow[b]{2}{*}{ Length of time } & \multicolumn{2}{|c|}{ Unadjusted } & \multirow[b]{2}{*}{$p$-Value } & \multicolumn{2}{|c|}{ Adjusted } & \multirow[b]{2}{*}{$p$-Value } \\
\hline & $\mathrm{HR}$ & $95 \% \mathrm{Cl}$ & & $H R$ & $95 \% \mathrm{Cl}$ & \\
\hline Registration time to patient left ED time & 1.13 & $0.87-1.45$ & 0.36 & 1.11 & $0.85-1.46$ & 0.44 \\
\hline $\begin{array}{l}\text { Physician initial assessment time to } \\
\text { disease-specific treatment administration time* }\end{array}$ & 1.79 & $1.29-2.48$ & 0.0005 & 1.50 & $1.05-2.15$ & 0.03 \\
\hline Length of time (minutes), & \multicolumn{2}{|c|}{ Lung POCUS group } & \multicolumn{2}{|c|}{ No lung POCUS group } & & \\
\hline median, Q1-O3 & \multicolumn{2}{|c|}{$n=81$} & \multicolumn{2}{|c|}{$n=243$} & $p$-Value * & \\
\hline Registration time to patient left ED time & 592.0 & $373-1,004$ & 626.0 & $405-1,212$ & 0.36 & \\
\hline $\begin{array}{l}\text { Physician initial assessment time to } \\
\text { disease-specific treatment administration time }\end{array}$ & 61.0 & $22-99$ & 92.0 & 49-203 & 0.0005 & \\
\hline
\end{tabular}

heart failure without a history of COPD received bronchodilators in the ED or by paramedics. ${ }^{21}$ This incidence is higher than our results; however, they included medication administered by paramedics.

\section{LIMITATIONS}

Our study had several limitations. First, the ability to collect patients' baseline characteristics was limited by the usual challenges posed by a health record review. ${ }^{22,23}$ For the exposure, patients who did not have archived lung POCUS or any such documentation were assigned to the no POCUS group. We could not determine the intended use of lung POCUS, i.e., whether it was for diagnosis, confirmation, or monitoring. The early diagnostic use of lung POCUS may have a different impact on the outcomes compared with its later use. It was not possible to know if the ED physician suspected a diagnosis of acute heart failure or COPD exacerbation at the time of the lung POCUS. Although ED physicians usually choose the patients who require lung POCUS based on history and physical exams, some physicians might have performed lung POCUS as a routine part of their exam. Second, the prevalence of using lung POCUS in our study population in the ED was low. This may have been due to various reasons including the perception of limited time or benefit to perform POCUS. Third, we were only able to follow patients who returned to the Ottawa Hospital or other clinics that sent clinical documents or letters to the Ottawa Hospital. Therefore, our results may underestimate the incidence of return visits. Fourth, we did not account for treatment administered before arrival to the ED by paramedics, which could have included intravenous fluids and bronchodilators. Therefore, the incidence of unnecessary treatment experienced by patients might be underestimated. Furthermore, due to the nonrandomized nature of the study, there is the risk of unknown and unmeasured confounders, although we did adjust for baseline imbalances. Possible unknown confounders include logistical factors such as availability of transport to allow patients to leave the department, or availability of consultants, which could all affect ED length of stay. Additionally, heart failure and COPD are complex disease processes for which long treatment periods are required, and where co-interventions are also likely to influence outcomes.

\section{CONCLUSIONS}

Although lung POCUS did not appear to decrease ED length of stay and time to disposition decision, we found that lung POCUS was associated with faster administration of disease-specific treatments for patients with suspected acute heart failure or COPD. The incidence of predefined adverse events was not significantly different. Our results suggest that lung POCUS improves the management of patients with suspected acute heart failure or COPD in the ED by specifically reducing the time to treatment. 


\section{SUPPLEMENTAL MATERIAL}

The supplemental material for this article can be found at https://doi.org/10.1017/cem.2019.499.

Acknowledgments: The authors thank all the staff of the Clinical Epidemiology Unit at the Department of Emergency Medicine in the Ottawa Hospital Research Institute.

Competing interests: None declared.

Financial support: No funding was received for this study.

Author contributions: S.N. conceived the idea. S.N., C.V., and M.T. structured the methods and the statistical analysis. M.W. and I.S. provided advice on the methodology and clinical implementation of this research. S.N. acquired the data and conducted analysis. M.N. contributed to the statistical analysis and interpretation. M.W. contributed in reviewing POCUS records for the interpretation and medical charts for the final diagnosis of acute heart failure. All authors critically reviewed the manuscript for its content and scientific accuracy.

\section{REFERENCES}

1. Kelly AM, Holdgate A, Keijzers G, et al. Epidemiology, prehospital care and outcomes of patients arriving by ambulance with dyspnoea: an observational study. Scand $\mathcal{7}$ Trauma Resusc Emerg Med 2016;24(1):113.

2. Hawkins NM, Petrie MC, Jhund PS, Chalmers GW, Dunn FG, McMurray JJ. Heart failure and chronic obstructive pulmonary disease: diagnostic pitfalls and epidemiology. Eur 7 Heart Fail 2009;11(2):130-9.

3. Ray P, Birolleau S, Lefort Y, et al. Acute respiratory failure in the elderly: etiology, emergency diagnosis and prognosis. Crit Care 2006;10(3):R82.

4. Wang CS, FitzGerald JM, Schulzer M, Mak E, Ayas NT. Does this dyspneic patient in the emergency department have congestive heart failure? $7 A M A$ 2005;294(15):1944-56.

5. Stiell IG, Clement CM, Aaron SD, et al. Clinical characteristics associated with adverse events in patients with exacerbation of chronic obstructive pulmonary disease: a prospective cohort study. Can Med Assoc 7 2014;186(6):E193-204.

6. Li L, Georgiou A, Vecellio E, et al. The effect of laboratory testing on emergency department length of stay: a multihospital longitudinal study applying a cross-classified random-effect modeling approach. Acad Emerg Med 2015;22(1):38-46.

7. Gill D, Galvin S, Ponsford M, et al. Laboratory sample turnaround times: do they cause delays in the ED? 7 Eval Clin Pract 2012;18(1):121-7.

8. Al Deeb M, Barbic S, Featherstone R, Dankoff J, Barbic D. Point-of-care ultrasonography for the diagnosis of acute cardiogenic pulmonary edema in patients presenting with acute dyspnea: a systematic review and meta-analysis. Acad Emerg Med 2014;21(8):843-52.
9. Martindale JL, Wakai A, Collins SP, et al. Diagnosing acute heart failure in the emergency department: a systematic review and meta-analysis. Acad Emerg Med 2016;23(3):223-42.

10. Mebazaa A, Yilmaz MB, Levy P, et al. Recommendations on pre-hospital \& early hospital management of acute heart failure: a consensus paper from the Heart Failure Association of the European Society of Cardiology, the European Society of Emergency Medicine and the Society of Academic Emergenc. Eur 7 Heart Fail 2015; 17:544-58.

11. Volpicelli G, Cardinale L, Garofalo G, Veltri A. Usefulness of lung ultrasound in the bedside distinction between pulmonary edema and exacerbation of COPD. Emerg Radiol 2008;15(3):145-51.

12. Volpicelli G, Mussa A, Garofalo G, et al. Bedside lung ultrasound in the assessment of alveolar-interstitial syndrome. Am 7 Emerg Med 2006;24(6):689-96.

13. Volpicelli G, Elbarbary M, Blaivas $M$, et al. International evidence-based recommendations for point-of-care lung ultrasound. Intensive Care Med 2012;38(4):577-91.

14. Van Walraven C, Davis D, Forster AJ, Wells GA. Timedependent bias was common in survival analyses published in leading clinical journals. 7 Clin Epidemiol 2004;57(7): 672-82.

15. Allison PD. Survival Analysis Using SAS: A Practical Guide. $2^{\text {nd }}$ ed. Cary, NC: SAS Institute Inc; 2010.

16. Blaivas M, Harwood RA, Lambert MJ. Decreasing length of stay with emergency ultrasound examination of the gallbladder. Acad Emerg Med 1999;6(10):1020-3.

17. Elikashvili I, Tay ET, Tsung JW. The effect of point-of-care ultrasonography on emergency department length of stay and computed tomography utilization in children with suspected appendicitis. Acad Emerg Med 2014; 21(2):163-70.

18. Chiem AT, Chan CHY, Ibrahim DY, et al. Pelvic ultrasonography and length of stay in the ED: an observational study. Am $\mathcal{F}$ Emerg Med 2014;32(12):1464-9.

19. Jones BP, Tay ET, Elikashvili I, et al. Feasibility and safety of substituting lung ultrasonography for chest radiography when diagnosing pneumonia in children: a randomized controlled trial. Chest 2016;150(1):131-8.

20. Matsue Y, Damman K, Voors AA, et al. Time-to-furosemide treatment and mortality in patients hospitalized with acute heart failure. 7 Am Coll Cardiol 2017;69(25):3042-51.

21. Singer AJ, Emerman C, Char DM, et al. Bronchodilator therapy in acute decompensated heart failure patients without a history of chronic obstructive pulmonary disease. Ann Emerg Med 2008;51(1):25-34.

22. Gilbert EH, Lowenstein SR, KozioI-McLain J, Barta DC, Steiner J. Chart reviews in emergency medicine research: where Are The Methods? Ann Emerg Med 1996; 27(3):305-8.

23. Kaji AH, Schriger D, Green S. Looking through the retrospectoscope: reducing bias in emergency medicine chart review studies. Ann Emerg Med 2014;64(3):292-8. 